\title{
Psychological intervention in breast cancer pathology
}

\author{
Diana-Antonia IORDACHESCU ${ }^{1}$, Corina-loana PAICA ${ }^{1}$, Elena-Otilia VLADISLAV ${ }^{1}$, Corina GICA ${ }^{2}$, \\ Radu BOTEZATU ${ }^{2,3}$, Anca Maria PANAITESCU ${ }^{2,3}$, Anca Marina CIOBANU ${ }^{2,3}$, \\ Gheorghe PELTECU ${ }^{2,3}$, Nicolae GICA ${ }^{2,3}$ \\ ${ }^{1}$ Faculty of Psychology and Educational Sciences, University of Bucharest, Romania \\ ${ }^{2}$ Filantropia Clinical Hospital, Bucharest, Romania \\ 3"Carol Davila" University of Medicine and Pharmacy, Bucharest, Romania
}

\begin{abstract}
Breast cancer is an extremely important public health issue for doctors. Health professionals draw attention to the importance of early detection of the disease, so that treatment becomes effective. The experience of the disease can be extremely shocking and devastating for women. Studies show that women diagnosed with breast cancer experienced prolonged emotional difficulties during the year following diagnosis.

This article proposes a review of some important methods and techniques used in the psychotherapy of breast cancer patients, such as: art therapy, cognitive-behavioral stress management, mindfulness-based stress reduction, acceptance and commitment therapy, compassion focused therapy and experiential psychotherapy.

This paper is a review based on information from the literature. The analysis was limited to articles and guides in English published between January 1 and June 1 2021, on PubMed, ScienceDirect and Google Scholar, using the following keywords: breast cancer, women, depression, distress, anxiety, mental health, psychotherapy, interventions, treatment. In this review we discuss the characteristics of breast cancer pathology, the psycho-emotional effects and the interventions needed to optimize quality of life.

The psychotherapeutic approaches presented draw attention to the fact that psychological interventions are part of the complementary procedures to the treatment in the pathology of breast cancer and can improve the quality of life of pacients
\end{abstract}

Keywords: breast cancer, pregnancy, mental health, psychotherapy, treatment

\section{BACKGROUND}

Breast cancer is an extremely important public health issue for doctors. The incidence of this pathology has increased greatly in the last decade, which highlights the concern of doctors, but also of women in general. According to the WHO [1], in 2020, there were 2.3 million women diagnosed with breast cancer and
685,000 deaths globally, being the most common cancer in women and the second most common cancer in general.

With progress in cancer disease diagnosis and treatment, the number of people healed continue to increase, but cancer survivors usually experience disease or treatment-related problems including both physiological and psychological problems and poorer quality 
of life. Currently, there is definite conclusion about the effectiveness of psychological interventions on cancer survivors' quality of life.

Doctors draw attention to the importance of early detection of the disease, so that treatment becomes effective. Breast cancer treatment consists of a combination of methods, from surgical removal, radiation therapy and drugs (hormone therapy, chemotherapy and/or targeted biological therapy) to treat microscopic cancer that has spread from the breast tumor through the blood. Such a treatment, which can prevent the growth and spread of cancer, saves lives. It is also known that breast cancer treatment affects women both physically and psycho-emotionally. The diagnosis represents for them an unexpected change in the trajectory of their lives.

Conventional healthcare mostly focuses on cancer survivors during or shortly after treatment [2] and may produce high cost and inconvenient access to health services due to the limitations of geography, time, and distance [3].

The psychological impact of breast cancer is well documented in studies. Given that this pathology is a leading cause of death among women, it is associated with emotional difficulties, personal, social and health losses [4]. Depression and anxiety are the most common emotional disorders in diagnosed women, which leads to decreased quality of life, work capacity and well-being.
Improved understanding of the types of distress and emotional difficulties that patients experience at the time of breast cancer diagnosis may reveal areas for intervention to improve quality of life and longterm outcomes [5].

The experience of the disease can be extremely disturbing and shocking for women. Studies show that women diagnosed with breast cancer experienced prolonged emotional difficulties during the year following diagnosis, but recent evidence suggests that individual differences affect how women respond to the diagnosis of breast cancer over time $[6,7]$. Researchers show that factors such as age, education, type of treatment and stage of the disease are associated with different levels of social maladaptation [8]. Parikh et al. [9] indicated that several women experience the following common symptoms following the diagnosis of breast cancer: fatigue, sexual difficulties, cognitive dysfunction, intrusive thoughts about the disease, anxiety, sadness, marital problems, communication difficulties about feelings of vulnerability and existential concerns about mortality.

Ludwigson et al. [5] identified two domains of distress to be higher on average and more frequently reported in patients with breast cancer: emotional and health distress. Additionally, social and practical distress, although lower on average than emotional and health distress, demonstrated wider variation in levels of distress. Furthermore, this study identified specific drivers of distress in each domain, including sadness

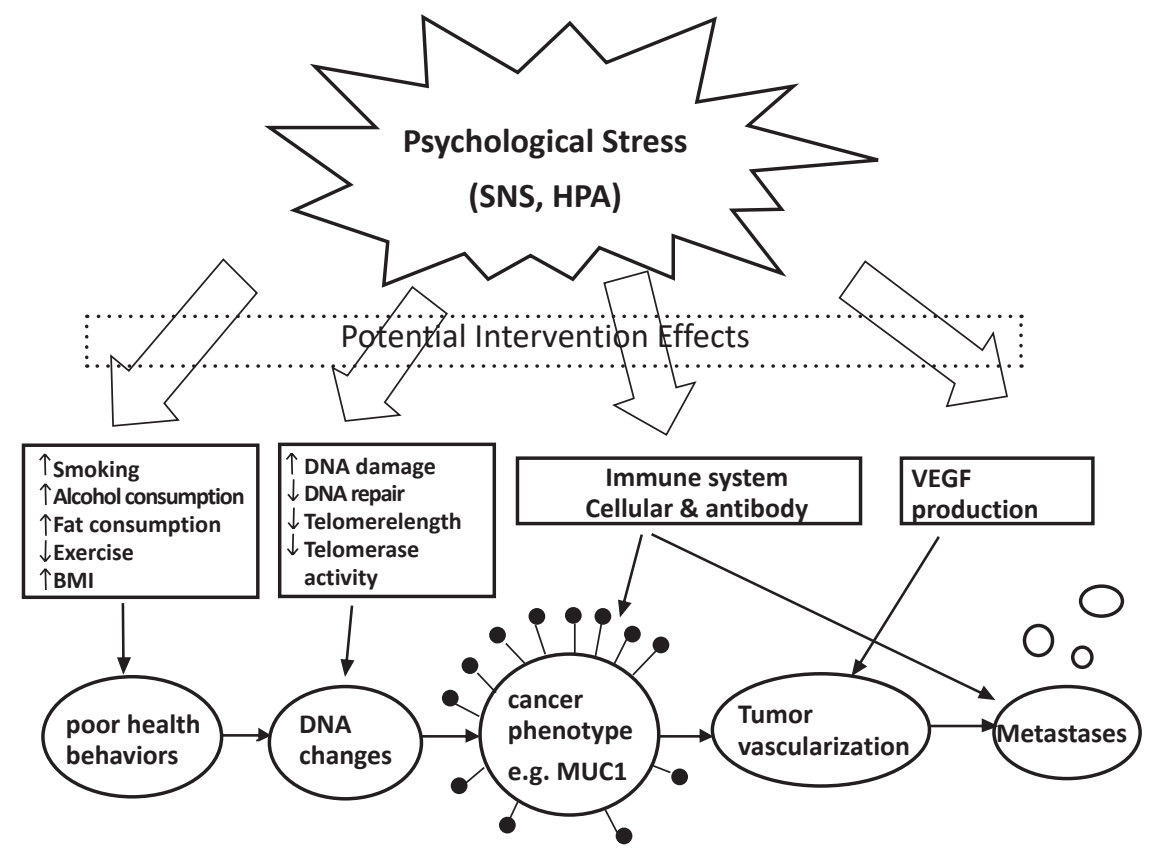

FIGURE 1. The development and progression of cancer and how psychological interventions might influence the process. SNS - synmpathetic nervous system; HPA - hypothalamus pituitary adrenal axis; BMI - body mass index; VEGF - vascular endothelial growth factor 
and depression, difficulty relating to friends and family, sleep disturbances, and financial concerns.

As illustrated in Figure 1, psychological distress can negatively influence multiple cancer relevant biological processes.

Psychological distress states are also associated with changes in gene function. Psychological stress is associated with increased DNA damage and poorer DNA repair $[10,11]$, and DNA repair pathways are important in the etiology of breast cancer.

\section{BREAST CANCER MANAGEMENT IN PREGNANCY}

Women who experience breast cancer during pregnancy are a challenge to the medical field. Studies estimate that $3 \%$ of women with breast cancer are pregnant at the time of diagnosis [12]. Opdahl et al. showed that nulliparous women have a higher risk of developing breast cancer than multiparous women [13]. Similarly, women who become pregnant with their first child after the age of 30 , have a slightly higher risk of developing breast cancer [14]. The diagnosis requires an approach adapted to both the needs of the mother and the risks that any treatment or medical intervention has on the fetus.

Data are limited in terms of long-term risks of anticancer treatment. Both the stage of the disease and the gestational age and the expected date of birth are significant factors in treatment [15]. Ring et al. [12] concluded in their review that in the first trimester of pregnancy the risks of harm to the fetus can be significant and thus treatment options are limited. Current studies on the short-term safety of breast cancer treatment in the second and third trimesters are reassuring.

Tesarova et al. [15] highlighted that chemotherapy can be safe in the second and third trimesters, but treatment needs to be stopped three weeks before the expected date of birth. At all stages, it is recommended that the patient to be followed by a multidisciplinary team that include the breast surgeon, oncologist, obstetricians, neonatologists and psychotherapist to ensure the best possible treatment for both mother and fetus. Non-ionizing examinations are used and preferred, and staging examinations that are performed may change the treatment of breast cancer during pregnancy [16].

Studies show that the most common pregnancy complications associated with chemotherapy are intrauterine growth restriction, prematurity, low birth weight and bone marrow toxicity [15]. Also, due to chemotherapy, the birth must be planned, and immediately after birth, breastfeeding is contraindicated.

Amant et al. [17] suggested that the further development of children born to women with cancer ap- pears to be similar to that of children of the same gestational age, regardless of in utero exposure to radiation or chemotherapy. However, data are limited in terms of cancer risk in children born to breast cancer patients.

To avoid diagnosis in an advanced stage of the disease, Durrani et al. [18] recommended genetic counseling to pregnant women, but also counseling is recommended to those who have had cancer in the past and want a pregnancy.

\section{PSYCHOLOGICAL INTERVENTIONS IN BREAST CANCER}

Authors such as Bardwell et al. [19] said that psychological factors have been found to predict overall quality of life (QoL) among breast cancer patients, rather than socio-demographic variables suggesting that psychosocial intervention can increase the QoL in this population.

Indeed, psychosocial intervention has been linked to improvements in QoL variables among breast cancer patients [20].

We propose a review of some important methods and techniques used in the psychotherapy of breast cancer patients.

Vella and Budd [21] evaluated the the effectiveness of a one-week residential withdrawal intervention that consists of photographic art therapy, group psychoanalytic therapy, and mind-body practices in reducing psychological distress and improving QoL for breast cancer patients. The results observed with the repeated MANOVA measures showed that these techniques lead to significant reductions in depression, anxiety and somatic stress and to sustained improvements in QoL and spiritual well-being.

Therefore, the authors conclude that art therapy in a group of cancer patients increases self-efficacy by acquiring new skills, reduces stress levels by providing an environment of concentration and is a basis for social support.

Antoni et al. [22] found that cognitive-behavioral stress management (CBSM) techniques correlate with significant reductions in moderate depression and are associated with increased optimism and benefit finding among breast cancer patients.

Mindfulness-based stress reduction (MBSR) is another type of psychotherapy that involves meditation and in which participants become more aware and accept negative emotions and cognitions without ruminating [23].

Regarding cancer patients, the evidence suggests that MBSR interventions can effectively improve QoL, sleep quality, mood and energy, while reducing perceptual stress, depression, anxiety and fear of cancer recurrence. 
Acceptance and commitment therapy (ACT) have shown promising effects on distress, QoL, and health behavior in cancer patients. Three non-randomized studies found that group-based ACT intervention among breast cancer patients reduced anxiety and depression [24], increased life expectancy [25], and promoted self-image [26] compared with that in a control group.

Concomitantly, a growing body of evidence demonstrate the importance of developing a self-compassionate attitude after a cancer diagnosis. Using Compassion Focused Therapy (CFT) [27] involves helping people to develop compassion towards the self, compassion to others, and openness in receiving compassion from others, especially in response to adverse situations such as having an illness.

In addition, optimism has protective effects, being associated with a better psychological adjustment in women facing breast cancer, being a predictor for the coping strategy "Accepting challenges", while pessimism is a predictor for the avoidance mechanism [6].

Li et al. [28] showed through their study that telehealth interventions are effective methods for improving the quality of life among cancer survivors. The most used methods are application-based intervention, website-based intervention and short-term telehealth intervention.

Most such interventions have included breast cancer survivors, and several well-designed control studies are needed to confirm the effects of telehealth interventions on the quality of life of cancer survivors.

In Romania, experiential psychotherapy is used in the psychological intervention of cancer patients. This includes expressive-creative techniques and methods that strengthen patients' selves. The experiential approach facilitates better self-knowledge and provides meaning to life experiences, including illness.

\section{CONCLUSIONS}

Breast cancer is generally a challenge for health professionals, but it is more difficult to manage in pregnant women due to the risks of treatments for the fetus. Doctors draw attention to the early detection of this pathology so that the treatment becomes effective. For women who want to become mothers or are pregnant, genetic counseling is recommended so as to avoid being diagnosed with advanced breast cancer.

Among the emotional disorders associated with breast cancer, depression and anxiety are the most common and lead to decreased quality of life, work capacity and well-being. The psychotherapeutic approaches presented in this article draw attention to the fact that psychological interventions are part of the complementary procedures to the treatment in the pathology of breast cancer that can improve the quality of life.

Due to the context of stress involving breast cancer pathology, it is necessary and recommended that patients participate in support groups under therapeutic guidance. It must be adapted to the needs and requirements of the patients throughout the treatment. Our recommendations also include continuing psychotherapy or participating in support groups after treatment, as many patients face fears of recurrence of the disease and problems with social maladaptation.

Conflict of interest: none declared Financial support: none declared

\section{REFERENCES}

1. World Health Organization. Available at: https://www.who.int/news-room/fact-sheets/ detail/breast-cancer (accesed: 12 June 2021)

2. van $\operatorname{der}$ Hout $A$, van Uden-Kraan CF, Holtmaat $\mathrm{K}$, et al. Role of eHealth application Oncokompas in supporting self-management of symptoms and health-related quality of life in cancer survivors: a randomised, controlled trial. Lancet Oncol. 2020;21:80-94.

3. Bradford NK, Caffery LJ, Smith AC Telehealth services in rural and remote Australia: a systematic review of models of care and factors influencing success and sustainability. RRH. 2016;16:3808.

4. Beutel ME, Weissflog G, Leuteritz K, et al. Efficacy of short-term psychodynamic psychotherapy (STPP) with depressed breast cancer patients: results of a randomized controlled multicenter trial. Ann Oncol. 2014;25:378-384
5. Ludwigson A, Huynh V, Bronsert M, et al. A screening tool identifies high distress in newly diagnosed breast cancer patients. Surgery. 2020;168:935-941.

6. Lam WW, Bonanno GA, Mancini AD, et al. Trajectories of psychological distress among Chinese women diagnosed with breast cancer. Psychooncology. 2010;19:1044-1051.

7. Deshields T, Tibbs T, Fan MY, Taylor M. Differences in patterns of depression after treatment for breast cancer. Psychooncology. 2006;15:398-406.

8. Helgeson VS, Snyder P, Seltman H. Psychological and physical adjustment to breast cancer over 4 years: identifying distinct trajectories of change. Health Psychol. 2004;23:3-15.

9. Parikh D, De leso P, Garvey $G$, et al. Post-traumatic stress disorder and post-traumatic growth in breast cancer patients - a systematic review. Asian Pac J Cancer Prev. 2015;16:641-646.

10. Flint MS, Baum A, Chambers WH, Jenkins FJ. Induction of DNA damage, alteration of DNA repair and transcriptional activation by stress hormones. Psychoneuroendocrinology. 2007;32:470-479.

11. Gidron Y, Russ K, Tissarchondou H, Warner $J$. The relation between psychological factors and DNA-damage: a critical review. Biol Psychol. 2006;72:291-304.

12. Ring AE, Smith IE, Ellis PA. Breast cancer and pregnancy. Ann Oncol. 2005;16:18551860.

13. Opdahl S, Alsaker MD, Janszky I, et al. Joint effects of nulliparity and other breast cancer risk factors. Br J Cancer. 2011;105:731-736.

14. Travis RC, Key TJ. Oestrogen exposure and breast cancer risk. Breast Cancer Res. 2003;5:239-247. 
15. Tesarova P, Pavlista D, Parizek A. Is It Possible to Personalize the Diagnosis and Treatment of Breast Cancer during Pregnancy?. J Pers Med. 2020;11:18.

16. Amant F, Loibl S, Neven P, Van Calsteren K. Breast cancer in pregnancy. Lancet. 2012;379:570-579.

17. Amant $F$, Vandenbroucke $T$, Verheecke $M$, et al. Pediatric Outcome after Maternal Cancer Diagnosed during Pregnancy. N Engl J Med. 2015;373:1824-1834.

18. Durrani S, Akbar S, Heena H. Breast Cancer During Pregnancy. Cureus. 2018;10:e2941.

19. Bardwell WA, Natarajan L, Dimsdale JE, et al. Objective cancer-related variables are not associated with depressive symptoms in women treated for early-stage breast cancer. J Clin Oncol. 2006;24:2420-2427.

20. Goodwin PJ. Support groups in advanced breast cancer. Cancer. 2005;104:2596-2601.
21. Vella EJ, Budd M. Pilot study: retreat intervention predicts improved quality of life and reduced psychological distress among breast cancer patients. Complement Ther Clin Pract. 2011;17:209-214.

22. Antoni MH, Lechner SC, Kazi A et al. How stress management improves quality of life after treatment for breast cancer. $J$ Consult Clin Psychol. 2006;74(6):1143-1152.

23. Carlson LE, Garland SN. Impact of mindfulness-based stress reduction (MBSR) on sleep, mood, stress and fatigue symptoms in cancer outpatients. Int J Behav Med. 2005;12:278-285.

24. Mohabbat-Bahar S, Maleki-Rizi F, Akbari ME, Moradi-Joo M. Effectiveness of group training based on acceptance and commitment therapy on anxiety and depression of women with breast cancer. Iran J Cancer Prev. 2015;8:71-76.
25. Ghasemi F, Dehghan F, Farnia V, et al. Effectiveness of Acceptance and Commitment Therapy on Life Expectancy of Female Cancer Patients at Tehran's Dehshpour Institute in 2015. Asian Pac J Cancer Prev. 2016;17:4113-4116.

26. Xue CC, Liu JE, Su YL et al. Development and preliminary verification of the group acceptance and commitment intervention program on body image of rehabilitative breast cancer survivors. Chinese Nursing Management. 2015;15:8-12.

27. Gilbert P. Introducing compassion focused therapy. Adv Psychiatr Treat. 2009;15:199-208.

28. Li J, Liu Y, Jiang J, Peng X, Hu X. Effect of telehealth interventions on quality of life in cancer survivors: A systematic review and meta-analysis of randomized controlled trials. IJNS. 2021:103970. 